Estudios sobre armas antiguas, arte militar $\mathrm{y}$ vida cultural en oriente y occidente

XXXVIII (2018), pp. 7-13

ISSN: 0436-029X

https://doi.org/10.3989/gladius.2018.01

\title{
LA LANZA DE ALEJANDRO EN GORTINA*
}

THE SPEAR OF ALEXANDER IN GORTINA

POR

\author{
Borja AnTEla-BernáRDEZ**
}

\section{RESUMEN - ABstract}

Una información recogida por Pausanias sobre la dedicatoria en Gortina de una punta de lanza que perteneció a Alejandro Magno permite considerar en detalle tanto la procedencia de la lanza como su valor curativo. El análisis nos remite, además, a las habilidades curativas de los Eácidas, y por ende, a las relaciones entre curación y poder en el mundo antiguo.

The information recorded by Pausanias about the dedication in Gortyn of a spear point that belonged to Alexander the Great allows us to consider in detail both the origin of this spear and its healing skill. The analysis refers us also to the healing habilities of the Aeacid dynasty and, after all, to the relationships between healing and rule in the Ancient World.

\section{Palabras Clave - Keywords}

Alejandro Magno, Gortina, armas curativas, poder curativo, Eácidas.

Alexander the Great, Gortyn, healing weapons, healing skills, Aeacids.

\section{Cómo CITAR este ARTículo / Citation}

Antela-Bernárdez, B. (2018): «La lanza de Alejandro en Gortina». Gladius, XXXVIII, 7-13. https://doi. org/10.3989/gladius.2018.01

En una de sus habituales descripciones, Pausanias informa que Alejandro Magno habría dedicado su lanza a Asclepio en el santuario que esta divinidad tenía en Gortina (Arcadia)ํ․

Yendo desde las fuentes del río, en primer lugar está el lugar de Márata y después de él Gortina, aldea en mi tiempo, pero anteriormente una ciudad. Allí hay un templo de Asclepio,

\footnotetext{
* Investigación desarrollada dentro del proyecto HAR2014-57096 El Impacto de la conquista de Alejandro (338279 a.C.), financiado por el Ministerio de Economía y Competitividad, y dirigido por B. Antela-Bernárdez y J. Vidal Palomino, y del Grup de Recerca Història del Conflicte a l'Antiguitat (2017SGR234), reconocido y financiado por la Generalitat de Catalunya. El autor agradece los consejos de A. I. Molina Marín y M. Mendoza Sanahuja, así como los de los referees anónimos, que han servido para mejorar de manera notable el resultado de esta investigación.

** Universitat Autònoma de Barcelona, Dept. de CC. de l'Antiguitat i l’E. M., Facultat de Lletres. borja.antela@uab. cat / ORCID iD: https://orcid.org/0000-0002-3118-3300.

1 Actual Atsícolo, entre Dimitsana y Karitaena, en un grupo de pequeñas colinas en la orilla derecha del río: Bölte, «Gortys» [5], RE VII, cols. 1672-1673. Cf. Herrero Ingelmo, 2008: 173 n. 191.
} 
de mármol pentélico, y una imagen de éste todavía sin barba, y otra de Higieia. Son obras de Escopas. Dicen también los del lugar que Alejandro, hijo de Filipo, ofrendó su coraza y su lanza a Asclepio y en mi tiempo todavía está la coraza y la punta de la lanza².

La noticia, que ha pasado desapercibida a la mayor parte de los historiadores de Alejan$\mathrm{dro}^{3}$, tiene cierto interés en cuanto a que evidencia la relación del conquistador con Asclepio. En una serie de trabajos recientes, hemos señalado diversos elementos de la asimilación de Alejandro con Asclepio 4 . No obstante, la cuestión de dedicatoria de esta lanza, junto con la coraza, nos parece merecedora de un análisis específico 5 .

Tal y como ha señalado la prof. M. M. Gabaldón, las armas de Alejandro debieron ser auténticas obras de arte, ejemplos magistrales de artesanía ${ }^{6}$, y sabemos por Plutarco incluso de sus autores:

Mandando esta respuesta a Parmenión, se colocó su casco; el resto de la armadura lo llevaba ya al salir de su tienda: una túnica siciliana, ceñida al cuerpo, y sobre ella una coraza doble, de lino, tomada como botín en Iso. En cuanto al casco, era de hierro pero resplandecía como pura plata, obra de Teófilo, y llevaba ajustada una gola igualmente de hierro, incrustada de piedras preciosas; llevaba también una espada admirable por su temple y liviandad, regalo del rey de Citio, pues se había habituado a servirse ante todo de espada en los combates. Llevaba también, abrochada a la espalda, una capa de una factura más esmerada que el resto de la armadura, pues era obra del antiguo Helicón, regalo de la ciudad de Rodas en señal de homenaje, y Alejandro la utilizaba también en las batallas?

Sorprende advertir que entre el equipo descrito para la batalla, en este caso Gaugamela, Alejandro no porte lanza alguna. De hecho, a lo largo de las fuentes podemos documentar cómo Alejandro aparece portando una lanza en muy pocas ocasiones: En el cruce del Helesponto, cuando clava su lanza antes de desembarcar para designar el territorio como ganado por la lanza ${ }^{8}$; en Gránico, cuando su lanza se rompe contra la coraza de Résaces ${ }^{9}$; en Tiro, cuando Alejandro lidera desde su barco el asedio, lanzando jabalinas a los de encima de la muralla ${ }^{10}$;

2 Paus. 8.28.1. Traducción de Herrero Ingelmo 2008.

3 La dedicatoria ha sido recogida por Gabaldón, 2004: 65-66. Asimismo, sobre dedicaciones de armas, vid. Baitinger, 2011.

4 Sierra y Antela-Bernárdez, 2016a: 397-417; Sierra y Antela-Bernárdez, 2016b: 115-140.

5 Sobre este tipo de prácticas, sigue siendo referencia obligada el trabajo de Rous 1902. Asimismo, sobre la pràctica de dedicación de corazas en santuarios, a lo largo del tiempo, en el mundo griego, vid. Graells i Fabregat 2017a; $2017 \mathrm{~b}$.

6 Gabaldón, 2004: 126.

7 Plu. Alex. 32, 8-12. Traducción de Bergua Cavero, 2007.

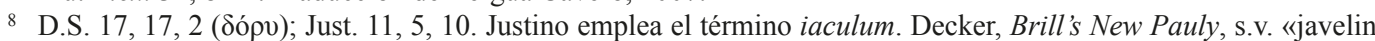
throwing» considera el iaculum equiparable la $\delta$ ó $\rho$, y en sí considerable como una javalina, y por tanto, pensada para su lanzamiento, tal y como de hecho sucede en el episodio narrado por Justino. Por otra parte, este mismo episodio tiene mucho que ver con el concepto de Asia como dorictetos chora: Vid. Mehl, 1980-1981; Hammond, 1988; Antela-Bernárdez, 2009; Antela-Bernárdez, 2011a; Antela-Bernárdez, 2011b; Antela-Bernárdez, 2016a.

9 Plu. Alex. 16, 8. El término empleado por Plutarco es dópv. Arriano $(1,15,5-6)$ también menciona la rotura, empleando el término $\delta$ ópv.

10 Curt. 4, 4, 11. El pasaje merece atención: Curcio dice que «a muchos que se defendían luchando desde lo alto de las murallas, los traspasó con su lanza; a algunos, incluso en combate cuerpo a cuerpo, los echó abajo empujándolos con su espada y con su escudo» (multos e muris propugnantes hasta transfixit, quosdam etiam comminus gladio clipeoque inpulsos praecipitavit: quippe turris, ex qua dimicabat, muris hostium propemodum cohaerebat). Se compaginan la lanza y la espada con el escudo, lo que evidencia que la lanza es un proyectil, algún tipo de jabalina. No obstante, el término de Curcio, hasta, es confuso. Traducción de Pejenaute Rubio, 1986. De hecho, Curcio menciona en diversas ocasiones una lanza de Alejandro $(4,6,24 ; 4,16,23 ;)$, aunque parece que ello responda a su narración y demuestre una cierta falta de criterio en los detalles militares, probablemente resultado de la realidad militar romana. 


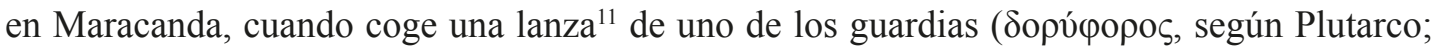
$\sigma \omega \mu \alpha \tau o \varphi v ́ \lambda \alpha \xi$ según Arriano) para atacar a $\mathrm{Clito}^{12}$; en Susa, cuando atraviesa con una sarisa a Oxatres el hijo de Abulites ${ }^{13}$; en su panoplia ante la embajada de $\mathrm{Nisa}^{14}$.

En este sentido, resulta un tanto útil, pese a los problemas que comporta el texto, recurrir a la descripción por Curcio de lo que podríamos considerar panoplia reglamentaria macedonia, tal y como aparece en relación con Corago para su lucha con Dioxipo:

En la mano izquierda, un escudo de bronce y una lanza denominada «sarisa»; en la derecha, una pica (lancea) y, en la cintura, una espada, como si fuera a combatir con varios enemigos a la vez ${ }^{15}$.

Sin embargo, si este es el equipo habitual para un falangita, no parece probable que el rey vaya equipado con estas armas. Y es que podríamos decir que sucede para la lanza de Alejandro exactamente lo contrario a lo que J. J. Moreno ha descrito ${ }^{16}$ para los escudos de la caballería de Filipo y Alejandro, que aparecen mencionados en las fuentes ${ }^{17}$, pero no en las representaciones iconográficas de la época. En el caso de la lanza ${ }^{18}$, conocemos representaciones de Alejandro kosmokrator obra de Apeles, donde aparece Alejandro asociado a la lanza

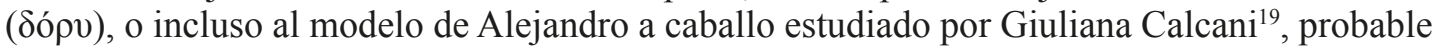
portador de una lanza. Frente a todo ello, sin embargo, lo único que podemos constatar es que, si bien Alejandro debió emplear la lanza como arma en sus acciones militares, y ello trascendió a la construcción de su iconografía, lo cierto es que no podemos advertir la existencia de «una» lanza, en un sentido especial, que haya podido ser específicamente objeto de dedicatoria a lo largo de la campaña ${ }^{20}$. Asimismo, vale la pena advertir del hecho que el término más habitual, como hemos visto, de nombrar este tipo de lanzas empleadas por Alejandro sería el de $\delta$ ó $v$.

Pasando a otra cuestión, resulta difícil señalar en qué momento de la campaña de Alejandro debió producirse la dedicatoria. Pausanias parece indicar que ello tuvo lugar antes del inicio de

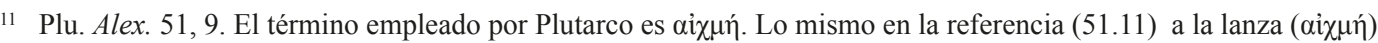

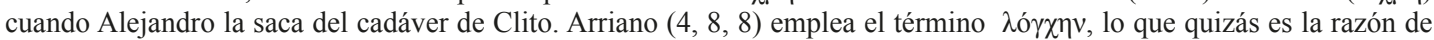
Guzmán Guerra, 1982, loc. cit., para traducir el término por el de sarisa. En la referencia de Clito a Eur. Andr. 693ss que

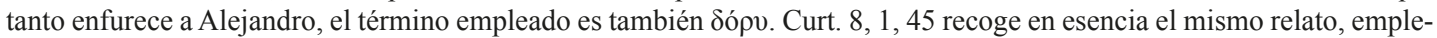
ando de nuevo el término hasta para denominar la lanza.

12 Parece poco probable suponer que la lanza de Alejandro dedicada en Gortina tenga que ver con el asesinato de Clito: las fuentes relacionan el episodio con Dioniso, no con Asclepio (Curt. VIII, 2, 6 y Plut. Alex. 13,4; 50; Antela-Bernárdez, 2017: 101-102). Por otra parte, existe una posible relación en el episodio entre Aquiles y Alejandro (King, 1987: 157), pero no parece que ello tenga que ver precisamente con la lanza en sí, sino solo con la v̋opıৎ de ambos. Por último, sobre el asesinato de Clito por Alejandro, vid. Carney, 1981.

13 Plu. Alex. 68, 7. El término empleado por Plutarco es sarisa ( $\sigma \alpha ́ p ı \sigma \alpha)$, lo que hace pensar que ésta no era un arma de Alejandro, por ser propia de la infantería (Arr. An. 1, 4, 2; 1, 6, 2; 3, 14, 3...), cuando Alejandro era, desde su ascenso al trono, el efectivo fundamental de la caballería. Sobre Alejandro en la infantería en Queronea, vid. Ma, 2008. Asimismo, Antela-Bernárdez, 2011c.

14 Arr. An. 5, 1, 4: «Esta embajada entró en la tienda donde Alejandro se encontraba, cansado y polvoriento del largo

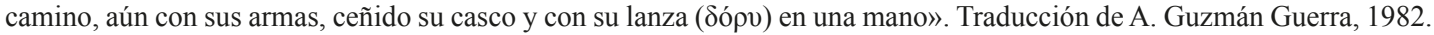

15 Curt. 9, 7. 19. Traducción de Pejenaute Rubio, 1986. D.S. 17, 100, 6 recoge también el episodio, mencionando lanza y espada.

16 Moreno, 2011: 539.

17 Especialmente en relación con Gránico: Cf. Hammond y Griffith, 1979: 417; Bosworth, 1996: 383-384.

18 Mucho se ha escrito sobre la lanza macedonia, la famosa sarissa: Mixter, 1992; Manti, 1992; Markle, 1999a; Markle, 1999b.

19 Calcani, 1989.

20 Pese a todo, las pocas menciones del uso de lanzas por Alejandro parece constatar que ésta no era quizás el arma más habitual, o al menos, por el hecho que se rompían, como en Gránico, su uso no implicaba ningún tipo de mención de interés. 
la expedición, justo después de la celebración del Sinedrión en Corinto en 335. Esta resulta, no obstante, una atribución difícil, aunque posible. En primer lugar, existe una distancia actual de alrededor de $140 \mathrm{~km}$ entre Corinto y Gortina, lo que habría llevado a Alejandro al corazón del Peloponeso, para una dedicatoria que por otra parte las Fuentes de Alejandro no recogen. Existen, claro, otros momentos específicos de la campaña, como algún tipo de agradecimiento al dios, que podrían responder a la dedicatoria, quizás en relación con la curación de alguna herida o enfermedad ${ }^{21}$, o quizás como agradecimiento por la protección recibida del dios en la guerra contra Persia ${ }^{22}$, o incluso como solicitud de depuración por el crimen cometido contra Clito. En este sentido, vale la pena recordar cómo Alejandro habría realizado dedicatorias en ausencia, sin estar en Grecia, como la de las famosas panoplias de Gránico a Atenea ${ }^{23}$.

La cuestión de la relación de Alejandro con el arte, sin embargo, aparece perfectamente planteada en el texto de Pausanias, quien nos informa tanto del autor de la estatua del supuesto Asclepio, como sería el magistral escultor Escopas, como del hecho que ésta no está barbada. Además, el conjunto escultórico estaría completado por una estatua de la salud. Todo ello nos permite garantizar, al menos, que cuanto se detalla en la descripción de Pausanias responde efectivamente a los tiempos de Alejandro. En este sentido, destaca la relación establecida por A. Stewart entre Escopas y la representación de pothos $^{24}$, encarnación de una emoción profundamente relacionada con Alejandro ${ }^{25}$.

Es asimismo curiosa la representación sin barba del dios. Estudios anteriores han analizado la compleja iconografía de Alejandro carente de barba ${ }^{26}$. Es posible que, como ha señalado Olaguer Feliu, la tendencia a representar dioses y reyes imberbes hubiese comenzado, sino incluso sido alentada, por el mismo Alejandro ${ }^{27}$. Ello nos lleva incluso a plantearnos si la estatua de Gortina se refiere a Asclepio o al mismo Alejandro asimilado al dios. De hecho, sabemos de una estatua a la Salud erigida en Atenas, como dedicatoria de Olimpia del Épiro, en algún momento a finales de la década del $330^{28}$, lo que sirve para garantizar no sólo la validez de la información de Pausanias, sino incluso para dotarla de mayor significado en los procesos de construcción de las imágenes legitimadoras del poder en los nuevos lenguajes de la realeza helenístico-macedonia. En este sentido, vale la pena recordar aquí que uno de los elementos de mayor valor en la relación iconográfica entre Alejandro y su madre es que ésta sirve de vínculo entre Alejandro y su imitado ancestro Aquiles ${ }^{29}$.

21 Interesante resulta la relación que en el texto subsiguiente de Pausanias se establece entre el río Gortinio, también llamado Lusio, y el Cidno, ambos de aguas muy frías (Paus. 8, 2-3), pues sabemos de la grave enfermedad de Alejandro en Cidno, que da lugar al famoso episodio del médico Filipo de Acarnania y el tópico de la traición de Parmenión. Sobre el episodio del río Cidno, vid. Sierra y Antela-Bernárdez, 2016a: 409-411. Sobre la relación entre los reyes macedonios y el cruce de ríos, vid. Molina Marín, 2016.

22 De entre diversas heridas de Alejandro, quizás las que tengan más opciones de estar relacionadas con la dedicatoria en Gortina sean las recibidas en Gránico o, más aun, las obtenidas en el asedio de la ciudad de los Malios, donde Alejandro recibe una herida de gravedad en el pulmón (a través de su coraza), y donde debió defenderse de numerosos enemigos, lo que quizás explicaría la dedicatoria de la lanza. Una última opción para la dedicatoria, en modo alguno descartable, es la de la dedicatoria posteriormente a la batalla de Gaugamela, cuando una lanza de Alejandro a punto estuvo de acabar con la vida de Dario; esta no sería una mala opción para la dedicatoria, ya que cronológicamente el episodio de la lanza en Gaugamela coincide aproximadamente con la revuelta espartana, con fuerte apoyo peloponesio, de Agis III. Pese a todo, cualquier respuesta es enormemente especulativa.

23 Gabaldón, 2004: 143, 166.

24 Arr. Indica, 20, 1-2. Cf. Ehrenberg, 1938: 52-61; Schachermeyr, 1973: 654ss; Stewart, 1993: 84 y nn. 47-48.

25 Stewart, 1993: 85, 118.

26 El trabajo fundamental es el de Alonso Troncoso, 2010. Asimismo, vid. Antela-Bernárdez, 2007: 92-93.

27 Olaguer-Feliu, 2000: 124-125.

28 Hyp. Euxen. 19. Asimismo, vid. Carney, 2006: 49, 95.

29 Sobre la relación entre Alejandro y Aquiles, vid. Cohen, 1995; Carlier, 2000; Carney, 2000; Antela-Bernárdez, 2007: 89-91; Antela-Bernárdez, 2016b: 246-248, con bibliografía actualizada. 
Durante su visita a Ilión, Alejandro tomo posesión de algunas de las armas de Aquiles. Sabemos explícitamente que Alejandro se apoderó de la coraza, y de hecho, parecen evidentes las coincidencias iconográficas entre la coraza de Alejandro representada en el Mosaico de Alejandro (probablemente sobre la batalla de Isos) atribuido a Apeles ${ }^{30}$ y la representación de Aquiles por el ceramista llamado el Pintor de Aquiles ${ }^{31}$. No obstante, es posible que además de la coraza, Alejandro hubiese tomado también otros elementos de la panoplia de Aquiles, en especial la lanza.

De la lanza de Aquiles sabemos que ésta habría sido, inicialmente, un regalo del centauro Quirón a Peleo. La lanza habría sido hecha de un fresno del Monte Pelión, lo que conecta de algún modo la lanza con las ninfas melíades, madres de los hombres de la Edad de Bronce y, según el más tardío Estacio, también de los arcadios. Posteriormente Peleo la habría cedido a su hijo Aquiles, quien la habría portado en su viaje a Troya, siendo él el único capaz de blan$\operatorname{dirla}^{32}$.

Asimismo, conocemos el carácter sanador de Aquiles por diversas informaciones ${ }^{33}$, el cual posiblemente fue además transmitido como componente de la familia real de los Eácidas del Épiro $^{34}$.Por Apolodoro sabemos también del episodio de Télefo, según el cual Aquiles habría herido a Télefo, y éste, incapaz de curar su herida, habría sido advertido por los augures de que la única cura residía en el mismo objeto que había causado la dolencia. Por ello, Télefo hubo de recurrir de nuevo a Aquiles, quien tocó de nuevo el lugar dañado con la lanza para que ésta pudiese sanar ${ }^{35}$. Ello, sin duda, permitió atribuir a la lanza dotes curativas, que sin duda habrían vinculado el arma a Asclepio. Por otra parte, esta relación de Aquiles con su lanza parece haber trascendido al arte, tal y como ha planteado Stewart al defender la identificación del modelo escultórico del doryphóros con el mismo Aquiles ${ }^{36}$.

Si volvemos a la noticia recogida por Pausanias sobre la dedicatoria ${ }^{37}$, podemos advertir cómo, más que la lanza, lo que se conserva (es decir, lo importante) parece haber sido la punta de la lanza ${ }^{38}$. Hemos visto también que resulta difícil discernir una sola lanza en la vida de Alejandro, una lanza que pudiésemos considerar como «la lanza» de Alejandro. Sin embargo, conocemos una lanza que estuvo en poder de Alejandro y podría perfectamente haber sido dedicada por él, como es la lanza de Aquiles que Alejandro obtiene en el templo de Heracles en Troya. No en vano, probablemente, la armadura de Aquiles que Alejandro obtiene en Ilión se encontraba en el templo de Heracles ${ }^{39}$, lo que conectaría la facultad curativa de la lanza de Aquiles tanto con Asclepio, patrón del santuario de Gortina, como con Gortis, el fundador del santuario, por vía de Heracles, con cuyo culto Gortis parece mantener cierta relación. El vínculo con Gortis puede establecerse por dos vías: primeramente, por la prueba de los pájaros de Estínfalo, que habitaban en el lugar del que era héroe epónimo Estínfalo el padre de Gortis; y en segundo lugar, por la violación por Heracles de Auge, hija de Aleo el hermano de Estínfalo

30 Del cual conocemos la copia encontrada en Pompeya, hoy conservada en el Museo Archeologico Nazionale di Napoli.

31 Cerámica ática (tipo b) de figuras rojas, actualmente en los Musei Vaticani: Cat. 16571.

Hom. Il. 16.140-144; Hom. Il. 19.387-391; Hom. Il. 22.133ff.

King, 1987: 9; Oller Guzmán, 2006: 240.

34 Cf. Antela-Bernárdez, 2017.

Hyginus, Fab. 101; Prop. ii.1.63ff.; Ovid, Ex Ponto ii.2.6; Plin. Nat. Hist. xxv.42, xxxiv.152.

Stewart, 1990: 160; Stewart, 1993: 162.

37 La dedicación de corazas por Alejandro ha recibido atención de los investigadores, sobre todo en relación con la dedicatoria a Atenas de las corazas de Gránico: cf. Squillace 1992-1994, con bibliografia.

38 Sobre las dedicaciones de puntas de lanza, aunque centrado en época cristina, resultan útiles las conclusiones del trabajo de Schulze-Dörrlamm 2011.

39 Anderson, 1928: 14. 
y heredero al trono de éste, en detrimento de sus hijos (entre ellos Gortis). Lo más interesante de esta violación, de hecho, es que el hijo de Heracles resultante no es otro que Télefo, el héroe herido por Aquiles y que sólo su lanza pudo curar. Con ello, el santuario tiene una clara relación con los Heráclidas y los Eácidas o descendientes de Aquiles, ambos linajes de Alejandro.

La última cuestión sea probablemente datar el momento de la dedicación por Alejandro. Si bien ello es, a la luz de los datos que poseemos, intensamente especulativo, considero que la mejor posibilidad tiene que ver con la revuelta de Agis, cuando algunos arcadios como los de Megalópolis se mantuvieron fieles a los macedonios. Es posible que la dedicatoria, por tanto, fuese posterior a la victoria de Antípatro contra los lacedemonios en 331, y sirviese de algún tipo de recompensa por su fidelidad.

\section{BIBLIOGRAFÍA}

Alonso Troncoso, V. (2010): «The Bearded King and the Beardless hero», D. Ogden y E. Carney (eds.), Philip II and Alexander the Great. Father and Son, Lives and Afterlives. Oxford, Oxford University Press: 12-24.

Anderson, A. R. (1928): «Heracles and his Successors». Harvard Studies in Classical Philology, 39: 7-58. https://doi.org/10.2307/310599

Antela-Bernárdez, B. (2007): «Alejandro Magno o la demostración de la divinidad». Faventia, 29: 6989.

Antela-Bernárdez, B. (2009): «Sucesión y victoria. Una aproximación a la guerra helenística». Gerión, 27: 161-177.

Antela-Bernárdez, B. (2011a): «Simply the best. Alexander's last words and the Macedonian kingship». Eirene, 47: 118-126.

Antela-Bernárdez, B. (2011b): «El carnero macedonio. La táctica de la falsa retirada en tiempos de Flipo y Alejandro», A. Espino (ed.), Nuevas fronteras de la historia de la guerra. Zaragoza, Pórtico: $29-48$.

Antela-Bernárdez, B. (2011c): «El día después de Queronea. La Liga de Corinto y el imperio macedonio sobre Grecia», E. Muñíz et al. (coord.), Grecia ante los imperios. Sevilla, Servicio de Publicaciones de la Universidad de Sevilla: 187-196.

Antela-Bernárdez, B. (2016a): «Poleis, Choras and Spaces, from civic to royal. Spaces in the cities over Macedonian rule from Alexander the Great to Seleucus I». Pyrenae, 47: 27-38.

Antela-Bernárdez, B. (2016b): «Like Gods among Men. The Use of Religion and Mythical Issues during Alexander's Campaing», K. Ulanowski (ed.), The Religious Aspects of War in the Ancient Near East, Greece and Rome. Leiden, Brill: 246-248.

Antela-Bernárdez, B. (2017): «Enfermedad, curación y poder. Los Eácidas taumatúrgicos», C. Sierra y B. Antela-Bernárdez (eds.), Historia y Medicina en la Antigüedad. Zaragoza, Pórtico: en prensa.

Baitinger, H. (2011): Waffenweihungen in griechischen Heiligtümern. Mainz, Monographien des Römisch-Germanischen Zentralmuseums 94.

Bergua Cavero, J. (2007): Plutarco: Vidas Paralelas VII. Madrid, Gredos.

Bosworth, A. B. (1996): Alejandro Magno. Madrid, Cambridge University Press.

Calcani, G. (1989): Cavalieri di Bronzo. La torna di Alessandro opera di Lisippo. Roma, L'Erma.

Carlier, P. (2000): «Homeric and Macedonian Kingship», R. Brock y S. Hodkinson, (eds.), Alternatives to Athens. Oxford, Oxford University Press: 259-268.

Carney, E. (1981): «The Death of Clitus». Greek, Roman and Byzantine Studies, 22: 149-160.

Carney, E. (2000): «Artifice and Alexander's History», A.B. Bosworth y E.J. Baynham (ed.), Alexander the Great in Fact and Fiction. Oxford: University Press: 263-285

Carney, E. (2006): Olympias, mother of Alexander the Great. London, Routledge.

Cohen, A. (1995): «Alexander and Achilles - Macedonians and Mycenaeans», J.B. Carter y S. P. Morris (eds.), The Ages of Homer: A Tribute to Emily Townsend Vermeule. Austin, The Texas University Press: 483-505.

Ehrenberg, V. (1938): Alexander the Great and the Greeks. Oxford, Oxford University Press. 
Gabaldón, M. M. (2004): Ritos de armas en la Edad del hierro: armamento y lugares de culto en el Mediterráneo y el mundo celta. Madrid, Ediciones Polifemo.

Graells i Fabregat, R. (2017a): «Las corazas incorruptas y la permanencia en exposición de algunas armas en santuarios (s. VI a.C. - II d.C.)». Ostraka, XXV: 53-66.

Graells i Fabregat, R. (2017b): «Armi mitiche, storiche e realinei santuari», R. Graells i Fabregat, F. Longo y G. Zuchtriegel (eds.), Le armi di Athena. Il santuario settentrionale di Poseidonia-Paestum. Napoli, Arte'm Editore: 147-162.

Guzmán Guerra, A. (1982): Arriano. Anábasis de Alejandro Magno. 2 vols. Madrid, Gredos.

Hammond, N.G. L. (1988): «The King and the land in the Macedonian Kingdom». The Classical Quaterly, 38: 382-391. https://doi.org/10.1017/s0009838800037022

Hammond, N. G. L. y Griffith, G. T. (1979): A History of Macedonia, vol. II. Oxford, Oxford University Press.

Herrero Ingelmo, M. C. (2008): Pausanias. Descripción de Grecia, libros VII-X. Madrid, Gredos.

King, H. (1987): Achilles. Paradigms of the epic hero from Homer to the Middle Ages. Berkeley: University of California Press.

Ma, J. (2008): «Chaironeia 338 BC». The Journal of Hellenic Studies, 128: 72-91.

Manti, P. (1992): «The Sarissa of the Macedonian Infantry». The Ancient World, 23: 32-42.

Markle, M. M. (1999a): «La sarisse macédonienne, la lance et l'équipement connexe», P. Brulé y J. Oulhen (eds.), La guerre en Grèce à l'époque classique. Rennes, Presses Universitaires de Rennes: 149-172.

Markle, M. M. (1999b): «Utilisation de la sarisse par Philippe et Alexandre de Macédoine», P. Brulé, J. Oulhen (eds.), La guerre en Grèce à l'époque classique. Rennes, Presses Universitaires de Rennes: 173-197.

Mehl, A. (1980-1981): «Dorictetos chora: Kritische Bemerkungen zum «Speererwerb» in poletik und Volkerrecht der Hellenistischen Epoche». Ancient Society, 11-12: 173-212.

Mixter, I. R. (1992): «The length of the Macedonian sarissa during the reigns of Philip II and Alexander the Great». The Ancient World, 23: 21-29.

Molina Marín, A. I. (2016): «The kings of Macedon and the Crossing of rivers», F. J. Gómez Espelosín y B. Antela-Bernárdez (eds.), El imperio de Alejandro. Aspectos geográficos e historiográficos. Alcalá de Henares, Servicio de Publicaciones de la Universidad de Alcalá de Henares: 51-74.

Moreno, J. J. (2011): Los orígenes del ejército de Filipo II y la falange macedonia. Tesis doctoral, Universidad Autónoma de Madrid.

Olaguer-Feliu, F. (2000): Alejandro Magno y el arte. Madrid, Encuentro.

Oller Guzmán, M. (2006): Origen y desarrollo del culto de Aquiles en la Antigüedad. Recogida y análisis de fuentes. Tesis doctoral, Universidad Autónoma de Barcelona.

Pejenaute Rubio, F. (1986): Quinto Curcio. Historia de Alejandro Magno. Madrid, Gredos.

Rouse, W. H. D. (1902): Greek votive offerings : an essay in the history of Greek religion. Cambridge, Cambridge University Press.

Schachermeyr, F. (1973): Alexander der Grosse. Viena, Osterreichischen Akademie der Wissenschaften.

Schulze-Dörrlamm, M. (2011): «Die Heilige Lanze in Wien. Die Frühgeschichte des karolingisch-ottonischen Herrschaftszeichens aus archäologischer Sicht». Jahrbuch des Römisch-Germanischen Zentralmuseums Mainz, 58: 707-742.

Sierra, C. y Antela-Bernárdez, B. (2016a): «Alejandro y la Anábasis de la medicina griega». Athenaeum, 104/2: 397-417.

Sierra, C. y Antela-Bernárdez, B. (2016b): «La divinización de Alejandro a través de la medicina». Quaderni Urbinati di Cultura Classica, 113/2: 115-140.

Squillace, G. (1992-1994): «Alessandro e l'offerta ad Atene di 300 panoplie». Miscellanea di Studi Storici, 9: 9-20.

Stewart, A. (1990): Greek Sculpture. An exploration. New Haven, Yale University Press.

Stewart, A. (1993): Faces of Power. Berkeley, University of California Press.

Recibido: 05-09-2017

Aceptado: 28-02-2018 